\title{
Migración: percepción infantil a partir de narraciones
}

\section{Migration: young people's perception from narratives}

Javier Zavala Rayas

Georgina Lozano Razo

María Dolores García Sánchez

Silvia del Carmen Miramontes Zapata

Juan Martín Sánchez Bautista

Universidad Autónoma de Zacatecas

\section{Resumen}

El objetivo de la presente investigación fue conocer y describir las percepciones que los infantes tienen de la migración internacional. La investigación se realizó en algunas localidades del estado de Zacatecas, pertenecientes a dos municipios con alta incidencia migratoria. Se trabajó con 483 niñas y niños, estudiantes de educación básica (primaria). Su usó la técnica del dibujo temático y se pidió que complementaran el dibujo con una historia sobre éste. Los resultados muestran que los participantes tienen conocimiento del fenómeno migratorio. Se encontraron dibujos e historias que giran en torno a algunas categorías que realizamos, de acuerdo con el contenido de las narraciones y dibujos, entre ellas, cumplir el sueño americano. Los participantes tienen perfectamente claro que la migración indocumentada conlleva peligros, por ejemplo, perecer al intentar cruzar el río; la falta de agua, alimento y exponerse a los animales venenosos al cruzar por el desierto; otro aspecto manifiesto es "la migra"; malos tratos hacia los indocumentados; además, en los dibujos e historias, existen indicios de dificultades en algunos aspectos emocionales, tristeza, sentimientos de abandono, desesperación, entre otros.

Palabras clave: migración, infantes, percepción, narrativa, metodología cualitativa.

Nota del autor

Javier Zavala Rayas, Unidad Académica de Docencia Superior, Universidad Autónoma de Zacatecas. Georgina Lozano Razo, María Dolores García Sánchez, Silvia del Carmen Miramontes Zapata y Juan Martín Sánchez Bautista, Unidad Académica de Psicología, Universidad Autónoma de Zacatecas.

La correspondencia en relación con este artículo debe dirigirse a Javier Zavala Rayas, Universidad Autónoma de Zacatecas, Jardín Juárez \#147, Col. Centro Histórico, C.P. 98000, Zacatecas, Zacatecas. Correo electrónico: jzavala@uaz.edu.mx 


\begin{abstract}
The objective of this research was to better understand and describe young people's perception regarding international migration. This research project was executed in different locations in the state of Zacatecas, in two municipalities with a high incidence of migration. The research subjects were 483 children attending elementary school. A thematic drawing technique was employed, in which subjects were asked to complete a drawing and tell a story about it. These drawings and stories generated a series of categories, among which were notions of the achievement of the American dream. The children have a clear understanding that undocumented migration is associated with certain dangers, such as drowning while crossing the river. Similarly, drawings are reminiscent of desert-like passages, including components such as lack of water, food, and the danger of venomous animals. Another category derived from the drawings and stories was related to the dangers of the "migra" and how young people describe the mistreatment of undocumented migrants. Additionally, the drawings and stories suggest emotional difficulties such as sadness, abandonment issues, anxiety, and others.
\end{abstract}

Keywords: migration, youth, perception, narrative, qualitative methodology.

Pretender siquiera hacer una descripción de la migración parece una ardua, difícil y compleja labor, ya que viene a ser una condición natural del ser humano, o como mencionan Sanz y Valenzuela (2016), "las dinámicas migratorias han sido sustancia de la historia humana... retratada por la movilidad de las personas, en todas las escalas y direcciones, tan continuas que pueden definirse como una práctica inherente a su existencia misma" (p. 15).

Olvera y Baca (2016), así como Calva (2019), indican que la migración suele realizarse en búsqueda de mejores condiciones de vida, y los motivos de ello serán tan diversos como el número de migrantes que existe en nuestro planeta. Estamos claros que el factor económico puede ser uno de los principales, pero no el único; se requiere un análisis desde diferentes perspectivas de las ciencias humanas y sociales, con un posible enfoque interdisciplinario, para tener una perspectiva comprensiva, $y$ potencialmente, establecer propuestas sólidas para analizar el fenómeno.

Por otro lado, Stefoni (2011), además de González y Soto (2015), mencionan que hasta hace pocos años, en la década de los noventa y en el 2000, principalmente, en el contexto mexicano se hacían investigaciones y/o se ponía mayor énfasis en las repercusiones económicas de las remesas que enviaban los migrantes -legales o no-, ya que el impacto de las mismas es enorme en la economía de México. Actualmente, y desde hace muchos años, representan la segunda fuente de ingresos, después del petróleo, y parece que pasarán a ser la primera, a reserva de las políticas que se establezcan por parte de la administración del presidente de Estados Unidos, en relación con 
los acuerdos comerciales y los migrantes. Lo anterior, en un panorama donde las políticas públicas van encaminadas a desaparecer la industria petrolera, donde la explotación de las riquezas del subsuelo mexicano pasa a empresas trasnacionales. Aunque hay quienes afirman que las remesas ya ocupan el primer lugar de ingreso de divisas en nuestro país (Mendoza, 2015; Las remesas, 2016).

A continuación se presentan algunas de las áreas en las que, desde una perspectiva psicosocial, puede analizarse el fenómeno migratorio y su relevancia: el impacto cultural identitario en adolescentes y jóvenes de retorno, que, en ocasiones, jamás habrían visitado México debido a las condiciones migratorias de los padres; estudiantes que viven en ciudades fronterizas de México y cursan los estudios en Estados Unidos; migrantes de retorno y la complejidad de su incursión en la educación en todos los niveles, con el inminente cambio en el sistema educativo y, algunas veces, sin conocer el idioma español; menores que migran sin los padres (Vilaboa, 2006; Villaseñor \& Moreno, 2006); además de condiciones sociales como la pobreza, el entorno familiar, y sus implicaciones para el fenómeno migratorio (Gallo, 2004; Rangel, 2008).

Algunos autores como Rodríguez (2008), se refieren a la relevancia del fenómeno migratorio internacional que, incluso, puede ser un elemento de seguridad nacional en algunos países, tanto receptores como emisores. Por otro lado, el análisis del impacto en la demografía es relevante, por ejemplo, en Zacatecas casi la tercera parte de la población vive en Estados Unidos de América, por lo cual se convierte en un estado binacional. Al 2010, de acuerdo con el Consejo Nacional de Población (CONAPO, 2012), y la Current Population Survey (2015), 572 mil zacatecanos estaban en Estados Unidos de América.

Se han estudiado las redes sociales y de apoyo que se emplean para lograr migrar, así como los lugares de origen y destino (Mendoza, 2003) como parte del fenómeno migratorio. Hacemos hincapié, también, en que la migración no debe ser sólo estadísticas, aunque relevantes, consideramos más importante enfatizar que es un fenómeno humano, que conlleva impacto a nivel psicológico y potencialmente repercutirá por el resto de la vida en el comportamiento de cada uno de los actores.

La migración, primordialmente realizada por varones solos, puede llevar a la desintegración familiar y a la implementación de una dinámica familiar distinta, forzada por las circunstancias, donde la figura paterna de la familia tradicional se desdibuja. Marchetti-Mercer (2012) y Falicov (2005), consideran y dan relevancia al hecho de que la migración no es un fenómeno individual, ya que afecta al migrante(s) y a su(s) familia(s). Por otro lado, Glick (2010) detalla algunas transformaciones que este fenómeno provoca en las relaciones intrafamiliares e, incluso, el trastrocamiento de valores, actitudes y la aculturación de las comunidades de origen. Gómez de León del Río y Guzmán (2006), 
además de McGuire y Martin (2007), describen algunos efectos psicológicos sobre miembros diversos de la familia o amigos cercanos, mientras que Díaz (2017), menciona algunas consecuencias en la salud mental de los infantes que se quedan cuando sus padres migran. D'Aubeterre (2002), Herrera y Carrillo (2009), señalan efectos poco favorecedores en las emociones a partir de la migración de los padres. Además de las repercusiones en la familia, pensemos en los efectos en la salud mental de las comunidades (Zavala, Luis, Lozano, García \& Robledo, 2008).

Según Piras (2016), los adolescentes que tienen padres migrantes, perciben $\mathrm{o}$ experimentan ambivalencia en los sentimientos, $\mathrm{y}$ tienen que reconfigurar sus emociones, ya que la ausencia o presencia no física, por las telecomunicaciones, no permite tener estabilidad emocional. Beck-Gernsheim (2003), hace referencia a que en los tiempos contemporáneos, aun cuando pueden ser confusos o relativamente no apegados a lo tradicional, la familia mantiene un reconocimiento como la construcción social donde se desarrollan y se forman los ciudadanos; al faltar el padre o la madre en la responsabilidad de la crianza y el cuidado, se genera confusión.

Paredes, Bravo y Calle (2004), hicieron una investigación con hijos de padres migrantes en Ecuador, y encontraron que se daban situaciones de maltrato infantil, enfermedades del tracto respiratorio, hipo crecimiento, problemas de nutrición, trastornos de conducta, ansiedad, depresión, baja autoestima, trastornos del sueño, enuresis, abuso sexual y trastornos del aprendizaje, esto último también lo reportaron Miranda-Vera, Mejía-Mayer y Díaz-Estrella (2018), además de patrones de conducta escolares inapropiados.

\section{Método}

El objetivo de la presente investigación fue describir la experiencia sobre el tema de la migración (padres migrantes) en niños de algunas localidades del estado de Zacatecas, estudiantes de educación básica (primaria). Estos resultados son parte de una investigación más amplia que implica investigación conjunta en otros estados de México -Hidalgo, Jalisco y Yucatán- y sólo se describirán los resultados del estado de Zacatecas.

La metodología utilizada es de carácter cualitativo, con base en el enfoque narrativo, ya que consideramos puede proporcionarnos más elementos para lograr la descripción de lo escrito por los participantes en la investigación. De acuerdo a Blanco (2011), esta perspectiva debe tener siempre presente que "la realidad" es heterogénea, múltiple, diversa, compleja y cabe la posibilidad de trascender campos disciplinarios. Bowman (2006), Webster y Mertova (2007), nos indican que lo narrativo nos lleva a la generación de textos reflexivos y, hasta cierto punto, experimentales, alejándose de la posibilidad de lo universal y de las leyes generales, empero, enfatizando lo concreto, individual y cotidiano, al contemplar la escritura como un método de análisis. Algunas de las 
preguntas de investigación que surgieron fueron: ¿saben qué es la migración? ¿Cómo perciben la migración los participantes? ¿Cómo les afecta?

\section{Participantes}

Se trabajó con 483 estudiantes de educación básica (primaria) de 9-12 años, de las localidades de Jerez (199 participantes, 120 mujeres y 79 hombres), Fresnillo (238 participantes, 150 mujeres y 88 hombres), Mariana (46 participantes, 26 mujeres y 20 hombres), del estado de Zacatecas. Cabe señalar que son localidades con alto índice migratorio hacia Estados Unidos de América.

\section{Técnica de recolección de datos}

Dibujo temático. Se eligió ésta técnica, ya que puede considerarse una actividad lúdica por parte de quienes participaron, y suele ser del agrado de niños y niñas de las edades que formaron parte de la investigación. Por otro lado, ocasionalmente suelen encontrarse algunas dificultades para contestar otro tipo de instrumentos psicológicos.

\section{Procedimiento}

Se acudió a las comunidades seleccionadas por sus características de tradición migratoria. Con antelación, se habían identificado las escuelas en las que se solicitó permiso a las autoridades educativas del plantel para realizar la investigación; se habló con directores y directora de las escuelas, se les explicó la actividad a realizar, a lo cual ellos accedieron sin dificultad y se envió el consentimiento informado a los padres y/o tutores de los infantes. Posteriormente, se trabajó con cada grupo, preguntándoles primero si sabían qué es la migración. Una vez que se verificó el entendimiento del término, se procedió a explicar la siguiente consigna: "van a hacer un dibujo sobre la migración, en la hoja que les vamos a dar, el dibujo lo pueden realizar como ustedes quieran, con colores o solo lápiz, usando toda la hoja o solo una parte. También van a anotar su edad y si son niños o niñas; así como su grupo. Al terminar el dibujo, en la parte de atrás de la hoja, van a escribir una historia sobre el dibujo que hicieron. Si hay alguna duda, pueden preguntar en cualquier momento. Gracias."

\section{Resultados y discusión}

A continuación se describen algunos dibujos e historias hechos por los niños sobre el tema de la migración, así como algunas categorías que fueron elaboradas con base en los elementos de los dibujos y las narraciones. Las categorías obtenidas son las siguientes: 1) separación de la familia, y 2) vicisitudes, subdividida ésta en "migra", desierto, sueño americano, falta de oportunidades laborales y muerte. A continuación, se procederá a ejemplificar dichas categorías.

\section{Separación de la familia}

Un niño de ocho años escribió: "este dibujo representa la familia y la migración, la migración es cuando se van de un lugar a otro y la familia cuando están todos juntos la migración es mala 
porque se ba un familiar que queremos". Por su parte, una niña de nueve años escribió: “el papá se va a Estados Unidos y su esposa su hija se ponen tristes. La migración es cuando alguna persona se va a otro país a veces es buena y a veses mala".

Además, tenemos que una niña escribió:

Mi papa se fue a Estados Unidos porque estaba muy dura la crisis y llo tenía 2 años y no lo e visto pero algun dia ba a regresar pero nos "miantiono" se fue de mogado por nos dise que aorita esta muy dura la crisis pero alla tiene ganas de venirse porque lla tiene mucho alla y es muy triste no tener aquí a sus familiares.

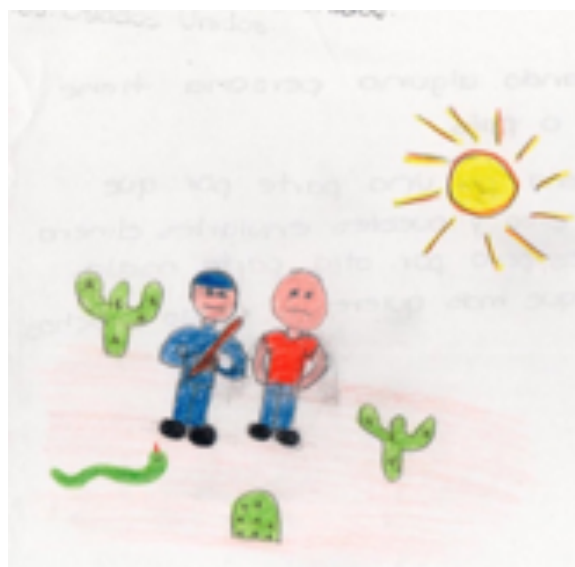

Figura 1. La migra (niño, 11 años)

Por otro lado, un niño comenta que:

Había una señora, un señor no tenian mucho dinero y el señor se fue al otro ldo dejando su hijo y su mujer cuando iba, lo agararon la señora llorando y llorando y su hija preguntaba y mi papá dónde esta mi papá y la señora no le contestaba, cuando cresio le dijo la verdad y ella se puso a llorar.

Además, encontramos que un infante escribió: "yo no tengo historia porque mis hermanos nunca han venido". Otro infante relató: la gente se va a los Estados Unidos por falta de recursos que aquí en México no ay por eso ay mucha migrasion a diferentes partes del mundo como mi tio que tiene 18 años en Notario California y no a venido en esos años, no lo conozco y tengo ganas de conocerlo". Catastróficamente tenemos: "mi primo Hugo se fue, deja sus familia en México por el tipo de trabajo, al poco tiempo se derrumbó el otel donde el vivia y murió. Ahora sus hijos están en $1^{\circ}$ y $2^{\circ}$ de secundaria y su madre murio de un infarto". La narración previa, podría ubicarse también en la subcategoría muerte.

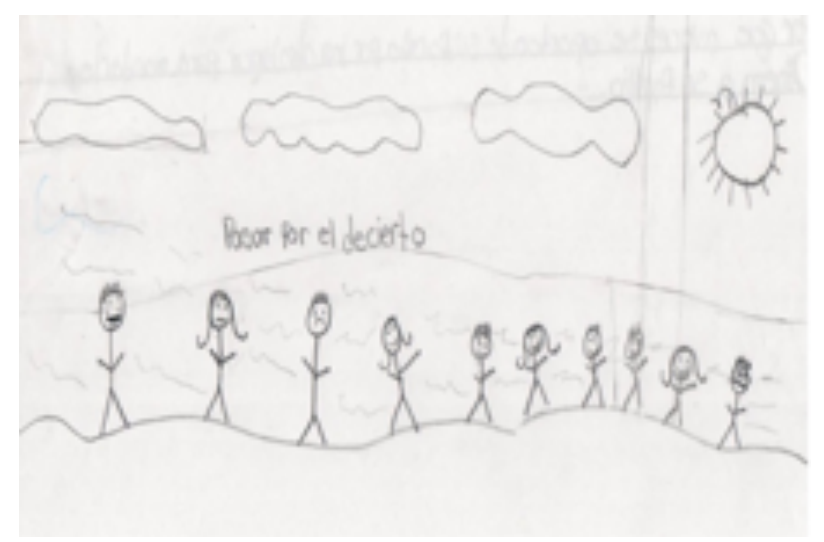

Figura 2. El desierto (niña, 10 años) 
Las narraciones nos describen aspectos, sobre todo, de tristeza y necesidad o ganas de establecer un contacto o conocer a sus familiares, ya que sus familiares migraron en algunos casos desde hace muchos años.

Imaginemos la zozobra de una familia que tiene referencia del peligro al cruzar la frontera de manera ilegal, ya sea por cruzar un río, brincar un muro o caminar por el desierto.

\section{Vicisitudes}

A este respecto, un niño de 12 años escribió: "porque cuando pasas el decierto te cansas y si se te caba el agua te puedes morir a la vez te puedes morir sin agua y alimento". Por otro lado, un niño de 10 años escribió: "tres señores que quieren crusar el desierto para llegar a Estados Unidos" (subcategoría desierto). También tenemos a otro niño de ocho años de edad que contó lo siguiente: "cuando se ban los papas a otros países, la migración para mi mala, porque pueden morirse" (subcategoría muerte). Otra niña de diez años relató: "la migración es cuando alguien quiere viajar a estados unidos por mojado y los atrapan a los matan es un poco mala y un poco buena y ellos quieren defender a su estado porque se van hacer una nueva vida y los que pasan o mueren por culpa de otros".

También encontramos, en relación a la subcategoría desierto, el siguiente relato: "yo divuje eso porque mis primos asi me contaron como pasaron ellos un primo mio me dijo que el paso por el decierto y que vido un elicotero $\mathrm{y}$ el yso un oyo en la tierra y se metio y el elicotero asuso pero no vido nada porque se avia escondido y tanvien que tomara agua puerca para sobrevivir". Además tenemos: "mi tío cruzo la frontera solo y en la dosch, por el desierto sin comida ni agua y no lo atrapo la policía". Como dato, Magallón (2008) reporta que desde la década de los noventa, se tienen al menos 450 muertes anuales registradas, acaecidas al pretender cruzar la frontera norte, sin contar con las víctimas que no se contabilizan y que suelen ser más elevadas que los reportes oficiales.

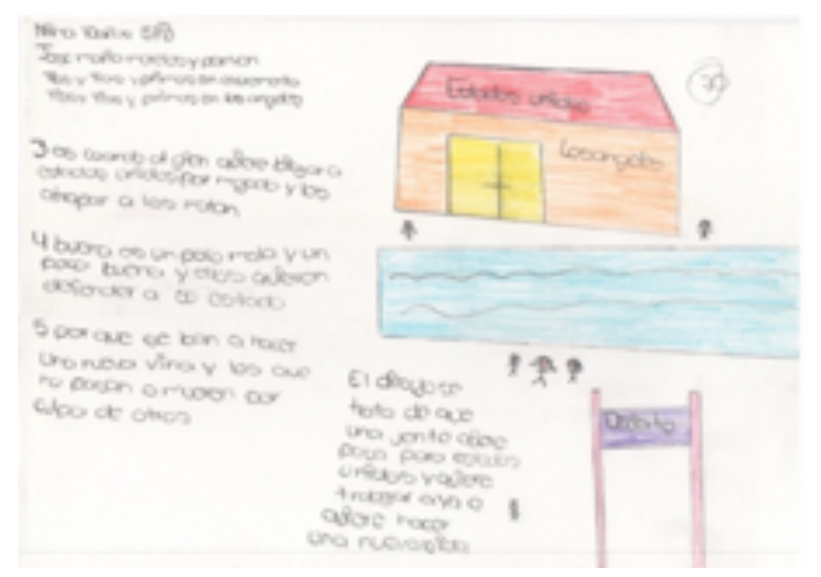

Figura 3. Una nueva vida (niña, 10 años) 
Encontramos en las narraciones que la muerte de quienes intentan cruzar de manera ilegal puede ser una constante, como es el caso de los siguientes relatos: "Yo lo puse porque los migrantes quieren pasar por el desierto pero la migra los matan y no llegan a su destino"; "Yo dibuje un señor que va pasar a Estado Unidos y pasaron unos aviones y les dispararon y se murió"; "Yo dibujé a un familiar mío que paso de mojado y que lo balacearon y se murió y llamaron a mi abuelita y lo velamos"; "Porque corren muchos riesgos como morir en el río o que klos asesinen"; "Cuando vas cruzando hay mucho peligros, también en las noticias dicen que matan a personas y las asesinan aunque sean inocentes y es muy peligroso por eso."

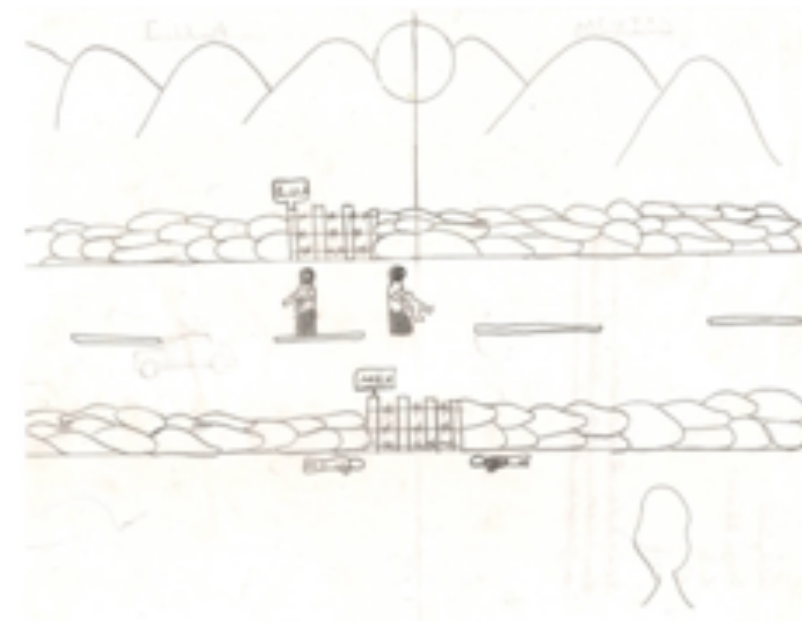

Figura 4. La muerte (niño, 12 años)

La "migra" es un elemento constante en las narraciones de los infantes, tienen referencia de ella: "Eataba cruzando y en eso la migra lo vio y lo trataron de matar"; "Mi papá me platicó que cruzó al otro lado y me contó que cruzó el rio le estaba disparando"; "Es injusta porque los policías les pegan, ellos no tiene la culpa, hasta las mamás se van y se llevan a sus hijos, pero a veces los dejan en la frontera. Está muy bien que las señoras les den de comer a los migrantes y tengan en donde descansar"; "Cuando el carro lleva carga y también gente y la baja al otro lado y tienen que pasar por el agua y si te ve la policía te meten a la carcel"; "Habia una vez tres compadres que querían cruzar la frontera por el rio bravo, primero paso Julio y cuando estaba pasando Pancho el de la migra le disparo."

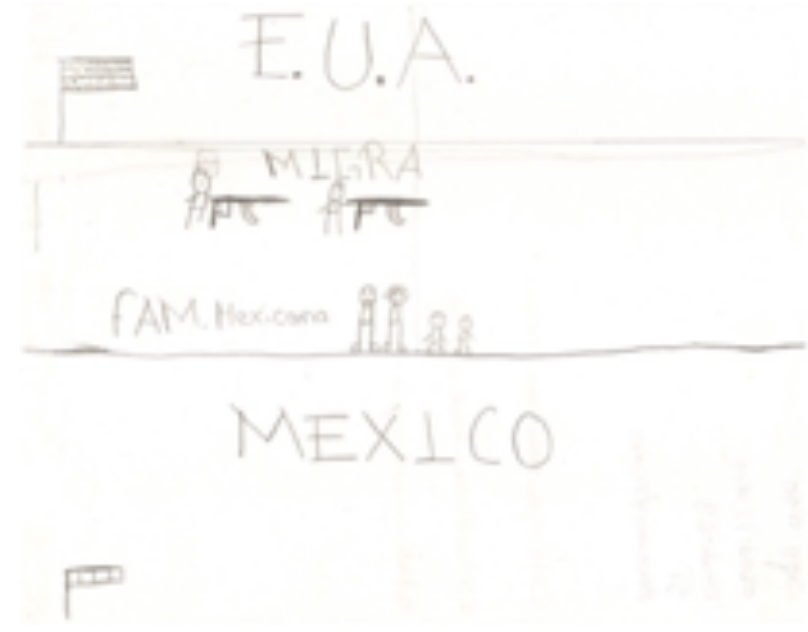

Figura 5. La frontera (niño, 10 años)

De acuerdo con Cárdenas, Gómez, Méndez y Yáñez (2011), así como Montañés y Moyano (2010), el análisis del fenómeno migratorio debe incorporar un abordaje en el cual se contemple que quienes migran, independientemente de las razones por las que lo hacen, son personas con relaciones familiares y sociales -amistades, escuela, entre otras-, y que la separación de esas familias nucleares e incluso extensas, conlleva 
un impacto, en lo general, negativo en las emociones, tanto de quienes se van como de los que se quedan.

De acuerdo con García, Lozano, Luis y Zavala (2011), para el 2004, los residentes en Estados Unidos de origen mexicano se estimaban en 26.6 millones, legales o no; aun con las restricciones impuestas para detener el ingreso de migrantes ilegales, la migración se mantiene constante, y debido a las carencias de opciones laborales mejor remuneradas, es difícil que se detenga.

Una niña de 10 años escribió en la subcategoría falta de oportunidades laborales: "es cuando no ay trabajo se ban al otro lado ay se puede encontrar para darle asu familia dinero". También tenemos: "la familia era una ves una familia un dia no tenian dinero para meter asu hijo na escuela y se fueron a estados unidos". El anterior relato pone de manifiesto que se tiene una clara percepción de por qué se emigra, la falta de recursos económicos y oportunidades de empleo en sus lugares de origen.

Diversos estudios mencionan que existe un impacto negativo en la salud psíquica de las poblaciones que padecen la migración (Aguilera \& Aldaz, 2003; Marroni, 2010; Ramos, 2010). En el ámbito de la niñez, algunos estudios muestran evidencia sobre la relación que existe entre la migración, la fractura de la familia y el bienestar psicoemocional; lamentablemente, el impacto negativo es generalizado (Flores \& Kaplan, 2009; McGuire \& Martin, 2007; Mummert, 2009).
López (2010) menciona que las investigaciones sobre el fenómeno migratorio en relación con los infantes como actores sociales de la migración son limitadas, a pesar de la relevancia de la situación, pues hay zonas en el país donde los infantes están inmersos en un ambiente, cuyos aspectos culturales, económicos, políticos, religiosos y psicológicos están impregnados de la migración y son parte cotidiana de la vida de quienes la viven, como lo ejemplifica la siguiente cita: “... jugaban como todos los niños a malos y buenos, a policías y ladrones, aunque en una variante local, pues unos eran 'emigrados' y los otros eran 'rinches', haciendo alusión probablemente a los rangers, del Programa Bracero" (p. 35).

Escobar, Hurtado, Pimentel, Rodríguez, y Santamaría (2008), mencionan que los hijos de migrantes que no emigran, suelen pasar a ser parte de las familias quebrantadas, puesto que la herida de sus integrantes es permanente y con la posibilidad de crecer, puesto que hay tensión, ya sea por el distanciamiento o la pérdida total de la persona que emigró y la probable presión

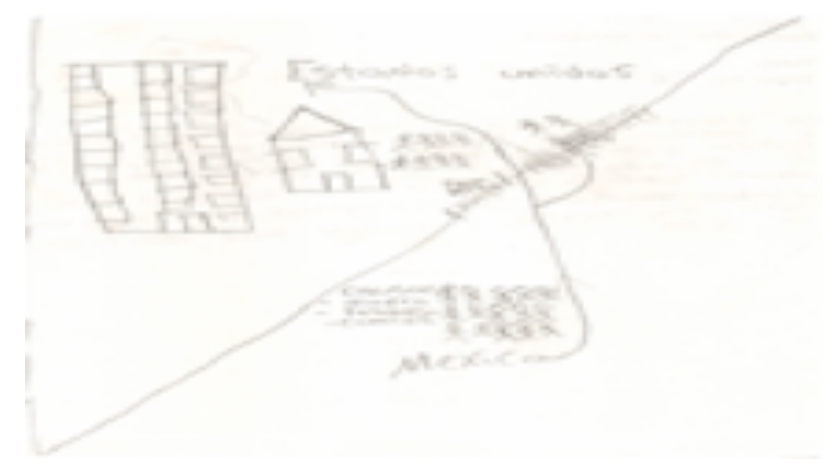

Figura 6. La frontera (niña, 10 años) 
social adquirida por las expectativas de una mejor calidad de vida, facilitada por el recurso económico que recibirá en el futuro; y los infantes tienen clara percepción de ello. Una niña de 10 años de edad mencionó: "que se van de mexico para ganar mas dinero y dejan a sus hijos esposa en mexico y ellos se van."

Una niña de 13 años escribió: "yo quisiera que toda mi familia estuviera junta pero todos mis abuelitos ya se murieron y mis tios están en el norte"; "La migración es cuando nuestro papas o tios viajan a otros lugares lejos... y si te ve la policía te mete a la carsel." Por otro lado, entre las dificultades psicosociales de los hijos de migrantes podría incluirse la desprotección, ésta se puede convertir en un problema al que quedan expuestos los niños y adolescentes. Elementos como la autoestima y quizá el sentido de pertenencia, podrían verse afectados por el fenómeno migratorio, además, la carencia de una figura de apoyo emocional que pueda compartir sus experiencias positivas o negativas, miedos o temores, así como sus dudas sobre diversos temas, lleva a estos niños y adolescentes a ser potenciales víctimas de grupos de personas que los amedrentan en la escuela y, en el peor de los casos, de la delincuencia organizada.

Por otro lado, en nuestros sistemas socioculturales latinoamericanos se da la solidaridad de la familia por consanguinidad, el apoyo en el cuidado y la crianza de los niños que tienen papá o mamá migrante, o ambos migrantes. Las tías y abuelas que se convierten en las "madres de aquí" (López \& Loaiza, 2010; Micolta \& Escobar, 2010). En la medida de lo posible, se subsana en el mejor de los casos la falta de la madre biológica, aunque esto no garantice que sea la mejor opción para el desarrollo emocional y afectivo de los infantes.

De acuerdo a Sotomayor-Peterson, y Montiel-Carbajal (2014), existe una ausencia de políticas públicas que satisfagan o estén a favor de las familias y los menores; los padres son los responsables en todos los aspectos de la población infantil en un entorno de escasa escolaridad, pobreza, falta de oportunidades laborales bien remuneradas, que permitan satisfacer las necesidades básicas, de recreación y esparcimiento, poca posibilidad de concluir o, al menos, iniciar estudios de nivel superior, por mencionar algunas carenacia y limitantes sociales. Estos entornos fomentan la violencia e inseguridad que actualmente vive la sociedad mexicana e impacta a todos los sectores sociales, con el creciente consumo de alcohol y fármacos en edades cada vez más tempranas.

Erazo, Esponda y Yaksic (2018), consideran que para implementar una política pública, en primera instancia, se debe reconocer presencia de una situación extraordinaria que amerite la posibilidad de desarrollar una política ex profeso. Para tal fin, hace falta investigación científica a cargo de grupos de expertos en la temática, que promuevan la necesidad de esas políticas públicas, además de responsables en la toma de decisiones en los poderes legislativos 
y ejecutivos, quienes lleven a cabo las acciones pertinentes para que dicha política sea una potencial realidad.

La situación actual de nuestro país, indica que falta apertura ante las necesidades de quienes realizan investigación en las ciencias sociales. Lo anterior hace más ardua la tarea de producir conocimiento que propicie el establecimiento de políticas encaminadas al sector infantil en el complejo tema de la migración. Debemos señalar y enfatizar, por considerarlo un punto álgido y hasta peligroso, la percepción del narcotráfico como algo natural o parte del proceso de migración. Al respecto, llamaron nuestra atención ciertos aspectos de las narraciones, que son "nuevos," pero no menos preocupantes. Es importante señalar que en años recientes, sobre todo 2016, 2017 y 2018, sin que haya disminuido ampliamente en 2019, según cifras oficiales del INEGI (2018), en Fresnillo y Jerez, cabeceras municipales en el Estado de Zacatecas, se tenía un elevado índice de inseguridad por el narcotráfico. Era común ver camionetas con personas armadas con rifles o ametralladoras y aunque actualmente es menos habitual, aún no desaparece ese tipo de eventos. Historias como las siguientes lo ejemplifican: "traficantes de marihuana cuando estaban pobres se los encontraron y les dijeron quieres dinero vamos a vender droga y vamos a ganar buena lana si ocupas dinero y te interesa vender toma 200 kilos y la mitad para ti y la mitad para $\mathrm{mi}$, te escondes para que la migra no te veo $\mathrm{i}$ no te meten a al cárcel no si no vendes droga y marihuana. Fin del cuento chistoso"; "Había tres señores que querían cruzar la frontera y el chapo Guzmán quería cruzar droga”; "Chapo se escapó de la cárcel se fue en un barco de policía es el más buscado de todo el país"; "El chapo migro a colima robo camino muchísima"; "El chapo es el más buscado de todo México lo atraparon los policías se les escapo por un túnel subterráneo la mariana lo vio le dieron un balazo en el pie no lo atraparon la historia continua". Es preocupante la relación migración-narcotráfico, ya que éste puede percibirse como una alternativa en el quehacer de las personas.

Por otro lado, nos pareció interesante que también aspectos de la migración internacional (de otros países, incluso continentes) se hiciera presente: "los migrantes de irác vienen a México para protegerse de la guerra otros a Europa y otros a Estados unidos de América”; "Que hay persona viaja para otros paise para mejor calidad de vida y que viene de muchas partes como un ondureño que iban para Estados unidos y se bajo por comida etren estaba parado y el se cayo y el tren le mocho una mano y un pie"; "Lo puse sobre una familia de egipto que migra hacia europa"; "Pues se trataba de un muchacho llamado Esteban que vivia en asia y no había mucho trabajo para el, como minero haci que migro a Estados Unidos, primero llego a México."

Por último, algunas narraciones sobre historias de "éxito" o de lograr el sueño americano, también estuvieron presentes: "que una familia migraron de México hacia estados 
unidos porque en México no havia tanto trabajo habúa escasos recursos y delincuencia y pasaron al rio lafrontera que divide México y estados unids y la familia crusando por tal de cruzar el rio bravo para cumplir su sueño Americano y para nir a buscar una nueva vida"; "Un día mi tia no tenia ni para comertenia unos aorros nada mas, haci decidio irse para Estados unidos y havi fue hizo sus maletas y se marcho al poco tiempo llama y nos dijo que ya tenia un empleo y ya camia muchio mejor"; "Era una vez un señor que era pobre y vivia en el campo el todos los dias iva a trabajar en su chilar el no comia ni carne ni nada solo sopa fijoles bueno todo lo normal pero ubo un dia que un amigo lo combido para irse al otro lado por falta de dinero y se fue cuando llego a alla su vida fue increible aunque todos los dias se lebantaba temprano tuvo todo lo que quiso. Fin"; "Era una ves un hombre que tenia una siembra pero era muy pobre un dia emigro a los estados unidos y le fue muy bien trabajo en un edificio y le fue exelente y su vida cambio y era un señor muy importante en la empresa y fue feliz para siempre. Fin"; "Mitio no tenia trabajo $\mathrm{y}$ entonces se fue a estados unidos tubo trabajo y le manda dineroami abuelita y pago todas sus deudas y aora vive felis."

El tema de la migración es del conocimiento generalizado en las localidades en las que se desarrolló la investigación. De igual forma, el tema económico es recurrente como uno de los motivos de la migración. Asimismo, encontramos historias relacionadas con quienes emigran; la migración no es exclusiva de los varones y los riesgos de cruzar hacia la frontera son diversos: el desierto, el muro y la migra, que incluso los pueden ser mortales. Por otro lado, tenemos que hay varias historias de éxito o donde se vive el sueño americano. Debemos señalar que el tema del narcotráfico nos hace reflexionar, ya que se encuentra inmerso en un fenómeno que habitualmente se consideraba de adolescentes, jóvenes y adultos; la droga puede ser un elemento que se convierte en motivo habitual para "migrar" o como un producto que debe llevarse más allá de la frontera.

\section{Discusión final}

La migración está presente en las localidades que formaron parte del estudio, se convirtió en algo cotidiano entre los miembros de esas comunidades. Sobresale el hecho de que procesos migratorios de otros países son del conocimiento de los infantes que participaron en el estudio, además de que, prácticamente, la totalidad de la población que participó tiene familiares de la familia nuclear o extensa que son migrantes.

Percepción sobre abandono por parte de quienes emigran, así como los cambios en los roles, sobre todo de las madres que se quedan, ya que se convierten en cabeza de familia ante la ausencia de la figura paterna, con un casi seguro impacto negativo en ellas como en los infantes, son aspectos imprescindibles para el establecimiento de políticas públicas con un enfoque en elementos psicológicos. 
Entre la población estudiantil suele ser habitual que haya decremento en las calificaciones de algún estudiante, pero poco se indaga si la migración puede ser parte de esa disminución en el rendimiento escolar, ya que éste puede verse afectado por sentimientos de abandono, tristeza, soledad, generados por la migración.

Entre las limitantes de este trabajo, tenemos que no estuvo orientado a la realización de diagnósticos, ni se emplearon otras herramientas como la entrevista individual, que permitieran aseverar la existencia de padecimientos psicológicos. De igual forma, sería adecuado continuar investigando en otros estados para conocer las diferencias y similitudes entre la niñez en torno al fenómeno migratorio.

Por otro lado, nos parece alarmante que, en las narraciones de los infantes, el tema del narcotráfico se manifieste con tal naturalidad y familiaridad. Al respecto, sugerimos que se ponga más atención a su influencia en el fenómeno de la migración y las interrelaciones que entre ellos van surgiendo a causa de las circunstancias que los alimentan y condicionan.

A partir de los dibujos y las historias que fueron producto de esta investigación, podemos aseverar que la migración se ha vuelto un aspecto habitual en los contextos investigados, tanto por las circunstancias que la propician como por las consecuencias que sufren quienes participan en este fenómeno. Algunos de los programas de atención gubernamental se centran en apoyo para establecer algún micronegocio de subsistencia, sobre todo para los migrantes de retorno, sin embargo, los aspectos emocionales y de salud mental se dejan de lado. Desde ámbitos académicos, es un fenómeno que requiere ser investigado en cuanto a los elementos afectivos que involucra, sus actores, y la complejidad que ello imprime al fenómeno.

Las narraciones de los niños reflejan cómo el fenómeno de la migración tiene relevancia por su impacto en el ámbito de la salud mental, en situaciones de alteración de emociones, ansiedad, depresión y algunos otros aspectos que repercuten en el ámbito psicológico. Ante esto, los autores planteamos la importancia de incluir dentro de las políticas públicas, aspectos psicológicos que incluyan a la población involucrada en la migración que, salvo en algunos aspectos, se encuentra totalmente desprotegida. De igual forma, incentivamos al gremio profesional interesado en el área a continuar con la investigación al respecto en diversos grupos etarios para ampliar el campo de conocimiento e intervención.

\section{Referencias}

Aguilera, R. y Aldaz, E. (octubre, 2003). La paternidad en el medio rural y la migración internacional, contradicciones de la masculinidad, la paternidad y la pareja. Trabajo presentado en el Coloquio Internacional Migración y Desarrollo, Zacatecas, Zacatecas. Recuperado de https:// www.colef.mx/emif/resultados/articulos/ circularidad $\% 20$ migratoria....pdf 
Blanco, M. (2011). Investigación narrativa: Una forma de construir conocimientos. Argumentos, 24(67), 135-156. Recuperado de http://www.redalyc.org/articulo. oa?id= 59521370007

Beck-Gernsheim, E. (2003). La reinvención de la familia. En busca de nuevas formas de convivencia. Barcelona: Paidós.

Bowman, W. (2006). Why Narrative? Why Now? Research Studies in Music Education, 27(1), 5-20.

Calva, J. L. (2019). Migración de mexicanos a Estados Unidos. Derechos y desarrollo. México: Juan Pablos Editores.

Cárdenas, M., Gómez, F., Méndez, L. y Yáñez, S. (2011). Reporte de los niveles de prejuicio sutil y manifiesto hacia los inmigrantes Bolivianos. Análisis de su relación con variables psicosociales. Psicoperspectivas, 10(1), 125-143. Recuperado de http://dx.doi. org/10.5027/psicoperspectivas-Vol10issue1-fulltext-134

Consejo Nacional de Población. (2012). Índice de Intensidad Migratoria: México-Estados Unidos 2010. México: Autor.

Current Population Survey. (2015). Annual Inmigration, Out Migration, Net Migration and Movers from Abroad for Regions: 1981-2017. Recuperado de https://www. census.gov/data/tables/2014/demo/ geographic-mobility/county-to-countymigration-2010-2014.html

D’Aubeterre, M. (2002). Migración transnacional, mujeres y reacomodos domésticos. En M. G. Marroni (Ed.), Con voz propia mujeres rurales en los noventa (pp. 225-297). México: Instituto de Ciencias Sociales y Humanidades de la Benemérita Universidad Autónoma de Puebla.

Díaz, M. (2017). Menores refugiados: impacto psicológico y salud mental. Apuntes de psicología, 35(2), 83-91. Recuperado de http://www.apuntesdepsicologia.es/index. $\mathrm{php} / \mathrm{revista} /$ article/view/662/482

Escobar, J., Hurtado, G., Pimentel, B., Rodríguez, V. y Santamaría, S. (2008). Los que se quedan una experiencia de migrantes. Revista científica electrónica de Psicología $U A E H, 6,1870-1882$.

Erazo, X., Esponda, J. y Yaksic, M. (2018). Migración y Derechos Humanos. Mediación Local Intercultural en el ámbito local. Autoctonía. Revista de Ciencias Sociales e Historia, 2(1), 159-162. doi: org/10.23854/ autoc.v2i1.62

Falicov, C. (2005). Emotional transnationalism and family identities. Family Process, 44(4), 399-406. doi: org/10.1111/j.15455300.2005.00068.x

Flores, L. y Kaplan, A. (2009). Addressing the mental health problems of border and immigrant youth. USA: National Center for Child Traumatic Stress.

Gallo, K. (2004). Niñez migrante en la frontera norte: Legislación y Procesos. México: Desarrollo Integral de la Familia y Fondo de las Naciones Unidas para la Infancia. 
García, M., Lozano, G., Luis, O. y Zavala, J. (2011). Familia y Migración. En J. Moral, J. Valdez y N. González (Eds.), Psicología y salud (pp. 253-276). México: CUMex.

Glick, J. (2010). Connecting complex processes: A decade of research on inmigrants families. Journal of Marriage and Family, 72(3), 498-515. doi: org/10.1111/j.17413737.2010.00715.x

Gómez de León del Río y Guzmán, J. (2006). The impact of absence: Families. Migration and family therapy in Ocotepec, México. American Family Therapy Academy, 2(1), 34-43.

González, A. y Soto, E. (2015). Migración y remesas en la región de las Haciendas, municipio de Santiago Ixcuintla, Nayarit: Un estudio exploratorio. Revista Mexicana sobre desarrollo local, 1(0), 27-37.

Herrera, G. y Carrillo, M. (2009). Transformaciones familiares en la experiencia migratoria ecuatoriana. Una mirada desde los contextos de salida. En G. Cortés y N. Miret (Eds.), Dialogues transatlantiques autour des migrations latinoaméricaines en Espagne. Reveu des Mélanges de la Casa Velásquez, 39(1), 97114. Recuperado de https://auth.openedition. org $/$ ?url=\%2Fproxy $\% 3$ Furl\%3Dhttp $\% 2$ $53 \mathrm{~A} \% 252 \mathrm{~F} \% 252 \mathrm{Fjournals}$. openedition. org\%252Fmcv\%252Fpdf\%252F591

Instituto Nacional de Estadística Geografía e Informática. (2018). Encuesta nacional de seguridad pública urbana (septiembre
2018). Recuperado de https://www.inegi.org. $\mathrm{mx} /$ contenidos/saladeprensa/boletines/2018/ ensu/ensu2018_10.pdf

Las remesas son ya el principal motor de divisas en México. (03 de febrero de 2016). El Diario. Recuperado de http://eldiariony. com/2016/02/03/las-remesas-son-ya-elprincipal-motor-de-divisas-en-mexico/

López, G. (2010). Niños, socialización y migración a Estados Unidos. En M. Ariza y A. Portes (Eds.), El país trasnacional, migración mexicana y cambio social a través de la frontera (pp. 545-570). México: Universidad Nacional Autónoma de México, Instituto Nacional de Migración y Miguel Ángel Porrúa.

López, L. y Loaiza, M. (2010). Padres o madres migrantes internacionales y su familia: Oportunidades y nuevos desafíos. Revista Latinoamericana de Ciencias Sociales Niñez y Juventud, 7(2), 837-860. Recuperado de http://www.redalyc.org/ html/773/77315614011/

Magallón, M. (2008). La migración de las ideas. En A. Santana (Ed.), Proyección global de la migración latinoamericana (pp. 61-89). México: Universidad Nacional Autónoma de México, Centro de Investigaciones sobre América Latina y el Caribe.

Marchetti-Mercer, M. (2012). Those easily forgotten: The impact of emigration on those left behind. Family Process, 51(3), 376-390. doi: org/10.1111/j.1545-5300.2012.01407.x 
Marroni, M. (2010). Mujer, madre y migrante: Los costos emocionales y psicosociales de una triple identidad. En L. Aresti (Ed.), Mujer y migración: los costos emocionales (pp. 133-143). México: Universidad Autónoma de Nuevo León, Universidad Autónoma de México y Universidad Michoacana de San Nicolás de Hidalgo.

McGuire, S. y Martin, K. (2007). Fractured migrant families. Paradoxes of hope and devastation. Family Community Health, 1(30), 178-188. doi: org/10.1097/01. FCH.0000277761.31913.f3

Mendoza, C. (Octubre, 2003). Circularidad migratoria, redes sociales y construcción de espacios trasnacionales en la migración México-Estados Unidos: aportes de una encuesta de flujos. Trabajo presentado en el Coloquio Internacional Migración $\mathrm{y}$ Desarrollo, Zacatecas, Zacatecas. Recuperado de https://www.colef.mx/emif/resultados/ articulos/circularidad $\% 20$ migratoria....pdf

Mendoza, G. (15 de septiembre, 2015). México recibe más dinero por paisanos que por petróleo. La Opinión. Recuperado de http:// laopinion.com/2015/09/15/mexico-recibemas-dinero-por-paisanos-que-por-petroleo/

Micolta, A. y Escobar, M. (2010). Si las abuelas se disponen a cuidar, madres y padres pueden emigrar. Revista Venezolana de Estudios de la Mujer, 15(35), 91-115. Recuperado de http://saber.ucv.ve/ojs/index.php/rev_vem/ article/view/2045/1947

Miranda-Vera, W., Mejía-Mayor, L. y DíazEstrella, M. (2018). Migración Parental: Incidencia en la conducta escolar en niños de educación general básica. Revista Psicología UNEMI, 2(2), 14-22. Recuperado de http:// ojs.unemi.edu.ec/index.php/faso-unemi/ article/view/697/593

Montañés, P. y Moyano, M. (2010). Violencia de género sobre inmigrantes en España: Un análisis psicosocial. Pensamiento psicológico, 2(6), 21-32.

Mummert, G. (2009). Siblings by telephone. Experiences of mexican children in long distance childrearing arrangements. Recuperado de www.highbeam.com/ DocPrint.aspx?DocId=1G1:222248458.

Olvera, J. y Baca, N. (2016). Continuidades y cambio en las migraciones de México a Estados Unidos. México: Universidad Autónoma del Estado de México.

Paredes, P., Bravo, L. y Calle, A. (2004). Impacto de la migración en la salud. Boletín de la Sociedad de Pediatría de Asturias, Cantabria, Castilla y León, (44), 137-149. Recuperado de http://www.sccalp.org/ boletin/189/BolPediatr2004_44_137-149. pdf

Piras, G. (2016). Emociones y migración, las vivencias emocionales de las hijas y los hijos que se quedan. Psicoperspectivas Individuo y Sociedad, 15(3), 67-77.

Ramos, M. (2010). Similitudes y diferencias en la salud psicofísica entre mujeres y hombres migrantes en Estados Unidos, implicaciones para las políticas de salud y programas de intervención social. En L. Aresti (Ed.), Mujer y migración: los costos emocionales (pp. 19-42). México: Universidad Autónoma de Nuevo León, Universidad Autónoma de 
México y Universidad Michoacana de San Nicolás de Hidalgo.

Rangel, G. (2008). Niñez migrante no acompañada en la frontera norte: Retos y desafíos. En G. Valdéz-Gardea (Ed.), Achicando futuros: Actores y lugares de la migración (pp. 147-160). México: El Colegio de Sonora.

Rodríguez, M. (2008). La migración internacional: Desafíos para América Latina y el Caribe. En A. Santana (Ed.), Proyección global de la migración latinoamericana (pp. 91-122). México: Universidad Nacional Autónoma de México, Centro de Investigaciones sobre América Latina y el Caribe.

Rosenfeld, M. J. y Tienda, M. (1999). Mexican Immigration, Occupational Niches, and Labor-Market Competition: Evidence from Los Angeles, Chicago and Atlanta, 19701990. En F. D. Bean y S. Bell-Rose (Eds.), Immigration and Opportunity: Race, Ethnicity, and Employment in the United States (pp 64-105). New York: Russell Sage Foundation.

Sanz, N. y Valenzuela, J. (2016). Migración y Cultura. México: El Colegio de la Frontera Norte.

Sotomayor-Peterson, M. y Montiel-Carbajal, M. (2014). Psychological and family WellBeing of unaccompanied mexican child migrants sent back from US border region of Sonora-Arizona. Hispanic Journal of Behaviour Science, 36(2), 111-123. doi:10.1177/0739986314523560

Stefoni, C. (2011). Migración, remesas y desarrollo. Estado del arte de la discusión y perspectivas. Polis: RevistaLatinoamericana, 30, 1-22. Recuperado de http://journals. openedition.org/polis/2389

Vilaboa, E. (2006). Caracterización de la niñez migrante en la frontera norte de México: Los casos de Tijuana y Nogales. México: Corredor Bilateral para la Protección de Derechos Humanos.

Villaseñor, B. y Moreno, J. (2006). La esperanza truncada: Menores deportados por la garita Mexicali-Calexico. Mexicali, BC: Albergue del Desierto, Centro de Reintegración Familiar de Menores Migrantes.

Webster, L. y Mertova, P. (2007). Using Narrative Inquiry as a Research Method. An introduction to using critical events narrative analysis in research on learning and teaching. New York: Routledge.

Zavala, J., Luis, O., Lozano, G., García, M. y Robledo, V. (2008). Migración: algunas consecuencias psicológicas en niños $\mathrm{y}$ adolescentes de dos municipios del Estado de Zacatecas. Investigación Científica, 4(2), 12-21.

Recibido: 13/03/2019

Revisado: 22/06/2019

Aceptado: 05/07/2019 